\title{
El Texto Guía como recurso didáctico en la enseñanza universitaria
}

\author{
The Study Guide as an Educational Resource in Higher Education \\ El Text Guia com a recurs didàctic en I'ensenyament universitari
}

\author{
José Luis Serrano Sánchez ${ }^{1, *(\bullet)}$, Víctor González-Calatayud ${ }^{2}{ }^{\circledR}$, María del Mar \\ Román-García ${ }^{10}$
}

1 | Departamento de Didáctica y Organización Escolar, Universidad de Murcia, Murcia, Murcia, España

2 | Departamento de Didáctica y Organización Escolar, Universidad Miguel Hernández, Elche, Comunidad

Valenciana, España

*Autor para correspondencia: jl.serranosanchez@um.es (José Luis Serrano Sánchez)

Recibido: 15/06/2020 | Aceptado: 30/09/2020 | Publicado: 25/12/2020

\section{Cómo citar:}

Serrano-Sánchez, J. L., González-Calatayud, V. y Román-García, M. M. (2020). El Texto Guía como recurso didáctico en la enseñanza universitaria. Research in Education and Learning Innovation Archives, 25,54-67. 10.7203/realia.25.17645

Copyright: El/La Autor/a. Open Access: Este es un artículo de acceso abierto distribuido bajo los términos de la licencia Creative Commons

Attribution-NoDerivatives 4.0 International licence (CC BY-ND 4.0)
RESUMEN: En este estudio se analiza el impacto de un material docente llamado Texto Guía en el proceso de enseñanza y aprendizaje dentro de una asignatura del Grado de Primaria de la Universidad de Murcia (España). Para ello, elaboramos un cuestionario ad hoc compuesto por 30 preguntas estilo Likert de 5 opciones, que fue validado mediante la técnica juicio de expertos. Las dimensiones de análisis fueron: el uso del Texto guía, su contenido y las actividades realizadas. Los resultados indican que los estudiantes valoran positivamente el uso, la estructura y la utilidad del Texto Guía para la preparación del examen, la realización de las tareas y la comprensión de los conceptos clave. Se observaron diferencias de género en relación con la utilidad de las rúbricas de evaluación puesto que las mujeres sí las encuentran útiles. Lo mismo sucede con las funciones de comprensión de conceptos y realización de tareas. Este trabajo puede contribuir al enriquecimiento de la convocatoria de Proyectos Textos Guía que publica cada año la Universidad de Murcia, ya que, por ejemplo, se ha analizado la estructura de este material, predefinida en la propia convocatoria.

PALABRAS CLAVE: educación superior; recursos didácticos; guía de fuentes de información

ABSTRACT: In this study we analyze how a teaching aid known as a Study Guide influences the teaching and learning process in a subject of the undergraduate degree in Elementary Education at the University of Murcia (Spain). To do so, we developed an ad hoc questionnaire comprising 30 five-option Likert-style questions. The questionnaire was then validated using the expert judgment technique. The dimensions of analysis were: use of the Study Guide, content of the Study Guide, and activities conducted. Our results show that the students valued the structure and usefulness of the Study Guide positively for preparing the examination, completing the course assignments and understanding key concepts. Gender differences were observed in relation to the usefulness of the evaluation rubrics since female students generally found them useful whereas male students did not. The result was the same when it came to the understanding of concepts and the completion of assignments. Our analysis of the structure of this material means that this research will also help strengthen the call for Study Guide projects published each year by the University of Murcia.

KEYWORDS: higher education; educational resources; guides to information sources

RESUM: Aquest estudi analitza l'impacte d'un material docent anomenat Texto Guía en el procés d'ensenyament i aprenentatge d'una assignatura del grau en Primària de la Universitat de Múrcia 
(Espanya). Vam elaborar un qüestionari ad hoc compost de 30 preguntes estil Likert de 5 opcions, que va ser validat mitjançant la tècnica de judici d'experts. Les dimensions d'anàlisi són: ús del text guia, del seu contingut i de les activitats realitzades. Els resultats indiquen que els estudiants valoren positivament l'ús, l'estructura i la utilitat del Texto Guía per a la preparació d'exàmens, la realització de tasques i la comprensió de conceptes clau. Hi ha diferències de gènere en relació amb la utilitat de les rúbriques d'avaluació, ja que les dones sí que les troben útils. Això mateix passa amb les funcions de comprensió de conceptes i la realització de tasques. Aquesta tasca també pot contribuir a l'enriquiment de la convocatòria de projectes Textos Guía que publica cada any la Universitat de Múrcia, ja que, per exemple, s'ha analitzat l'estructura d'aquest material, predefinida en la convocatòria mateixa.

KEYWORDS: educació superior; recursos didàctics; guies de fonts d'informació

\section{Notas de aplicación práctica}

\section{Qué se sabe sobre este tema}

- Los estudiantes universitarios suelen mostrar dificultades para convertir la información contenida en textos académicos a conocimientos aplicables.

- El profesorado universitario tiene altas expectativas en relación con las estrategias necesarias que -supuestamente- los estudiantes deberían tener para realizar lecturas con mayor rigor y profundidad analítica.

- Las dificultades que tienen los estudiantes para buscar y seleccionar la información que necesitan para generar nuevos conocimientos.

\section{Qué aporta este trabajo}

- Creación de un manual docente (Texto Guía) más ajustado al perfil del estudiante universitario actual en el marco de una metodología activa basada -principalmente- en el aprendizaje por tareas. Además de un cuestionario validado para valorar su utilidad.

- Evidencias para mejorar la estructura del Texto Guía como recurso educativo en la enseñanza universitaria.

- La comprensión de los contenidos seleccionados por el profesor sigue siendo un aspecto valorado positivamente por los estudiantes. Siendo el elemento que más les preocupa.

\section{Implicaciones para la práctica y/o política}

- El cuestionario validado podrá ser utilizado en otras asignaturas en las que se utilicen Textos Guías o manuales docentes equivalentes para que los estudiantes puedan valorar su utilidad.

- Los resultados permiten influir en la estructura del diseño de los Proyectos Textos Guía propuesta en la convocatoria anual que publica la Universidad de Murcia.

\section{INTRODUCCIÓN}

La bibliografía es una fuente indispensable de conocimiento que otorga al alumnado las bases teóricas que le permiten analizar las cuestiones empíricas relacionadas con la disciplina en la que se está trabajando. Por lo tanto, el uso de la bibliografía en el ámbito académico se convierte en una necesidad a lo largo de todo el proceso de formación del alumnado universitario. En muchas ocasiones los recursos bibliográficos que incluimos en nuestras asignaturas consisten en textos científicos, como pueden ser artículos o capítulos de libros, los cuales utilizan un lenguaje dirigido más a profesionales en el ámbito que a estudiantes (Carlino, 2003). Por lo tanto, en este tipo de textos se utilizan unas categorías conceptuales, líneas de pensamiento y polémicas internas del campo que dificultan la comprensión de dichos textos por parte del alumnado (Marucco, 2011). Las dificultades para los estudiantes suelen aumentar considerablemente ante las expectativas que el profesorado tiene hacia ellos, puesto que se pretende evaluar al alumnado en función de un determinado modelo de lectura -saber identificar la postura del autor, reconocimiento de las posturas y argumentos, poniendo lo leído en relación 
con otros textos, etc.- que el alumno no tiene interiorizado (Carlino, 2003; Estienne y Carlino, 2004). Es decir, se les pide una lectura con mayor rigor y profundidad analítica (L. B. Peña, 2008) en vez de una lectura superficial. En los niveles previos a la universidad la lectura se enmarca en espacios académicos formales, limitando su uso a tareas muy concretas de cada asignatura (F. J. Peña, 2011). Sin embargo, esas estrategias de procesamiento de la información más general no son fácilmente extrapolables a una disciplina en particular (Vázquez, 2005), dando lugar a un nuevo tipo de alfabetización que se ha denominado "alfabetismo académico" (Marín, 2006).

Si a esta problemática se le añade que, nos encontramos en un mundo de información cambiante que irremediablemente tiene como consecuencia que la información sea inabarcable y la dificultad que tiene la búsqueda de información más específica, nos puede llevar a una situación en la que el alumnado no tiene los recursos suficientes para encontrar la información que necesita (Henry, 2006). Con toda probabilidad, esta situación acabará afectando al estudiante en el desarrollo del resto de competencias.

Como algunos autores destacan (Henry, 2006; López-Flamarique, 2017), en muchas ocasiones los alumnos no diseñan un plan óptimo de búsqueda de información, lo cual les suele conducir a la desorientación. Comenzamos a tener evidencias de que los estudiantes universitarios no suelen tener la competencia suficiente para buscar información de calidad (Egaña, Bidegain, y Zuberogoitia, 2013) puesto que las estrategias de búsqueda suelen ser deficientes, utilizando Google y Wikipedia como principales y únicas herramientas de búsqueda (Prendes, Solano, Serrano, González, y Román, 2018). En este sentido, el problema se agudiza cuando los datos nos indican que los estudiantes se consideran capaces de seleccionar información apropiada que encuentran en la Web (Gutiérrez y Serrano, 2016), pero muestran indiferencia generalizada para contrastar la información o de acceder solamente a páginas con criterios de calidad científica (Gutierrez, Lopéz, Serrano, y Solano, 2017), lo cual es una incongruencia importante.

Aunque se debe abordar con urgencia el desarrollo del área de acceso a la información de la competencia digital del alumnado para paliar estos déficits, existen otras estrategias que pueden ser, cuanto menos, complementarias. Por ejemplo, un modo de poder evitar esta problemática es la creación de un manual o Texto Guía que le sirva al alumno como canal para comprender otros textos y su vinculación con el resto de las partes de la asignatura (tareas, evaluación y competencias). No se trata de servir el libro como de un menú preestablecido, sino más bien como una carta más abierta en la que deben ir seleccionando aquello que necesitan. Dicho de otro modo, un punto de partida más amigable para acabar profundizando en el contenido de la materia.

Sin embargo, este recurso educativo ofrece otras posibilidades tanto para los docentes como para los estudiantes universitarios. Va mucho más allá de una especie de resumen de contenidos que facilite al estudiante el estudio de estos. Ésta es precisamente la principal finalidad de este artículo, mostrar a toda la comunidad universitaria en qué consiste este recurso, cómo se está utilizando en la Universidad de Murcia y la valoración de algunos de los futuros docentes de Primaria tras su utilización en el marco de la asignatura "Aplicaciones sociales de las TIC para la Escuela y el tiempo libre” del Grado en Educación Primaria de la citada universidad.

\section{EL TEXTO GUÍA EN LA UNIVERSIDAD DE MURCIA}

La Universidad de Murcia, a través del Centro de Formación y Desarrollo Profesional convocó un concurso para la selección de proyectos de docencia universitaria Textos Guía. El objetivo de la convocatoria es ofrecer al profesorado de la Universidad de Murcia la posibilidad de reflejar en un Texto Guía (manual docente en formato libro) la planificación y concreción de una asignatura. 
Estos proyectos de docencia universitaria debían presentar una estructura específica y predefinida que sirviera como recurso para la orientación en el proceso de enseñanza y aprendizaje. De esta manera, se apoya la realización de materiales de aprendizaje para el alumnado de la Universidad de Murcia y se ofrece al profesorado la posibilidad de publicar en formato libro sus materiales de enseñanza.

Teniendo en cuenta la percepción de algunos estudiantes universitarios, mostramos en este trabajo el impacto del Texto Guía que diseñamos para la asignatura "Aplicaciones sociales de las TIC para la Escuela y el tiempo libre” (Serrano, Román, González, y Prendes, 2016). Con la creación de este material docente hemos permitido a los estudiantes de la citada asignatura encontrar una relación directa de las competencias, núcleos temáticos, contenidos, tareas a realizar, metodología a seguir, resultados de aprendizaje, duración, así como criterios y rúbrica de evaluación por cada una de las actividades. La principal finalidad es mejorar la comprensión de la relación existente entre todos los aspectos clave que figuran en la ficha y en la guía docente de la asignatura, puesto que se tiene un mayor nivel de concreción.

El Texto Guía tuvo un papel protagonista en la asignatura. Durante su planificación, el equipo docente reflexionó sobre la relación entre las competencias y los resultados de aprendizaje esperados (según la memoria verificada de la ANECA y la Guía Docente) con los contenidos, la metodología, las actividades, la evaluación y la bibliografía. Durante la asignatura, los estudiantes utilizaron el Texto Guía como un recurso básico para seguir la materia.

La propia convocatoria de Textos Guía de la Universidad de Murcia marcaba la estructura a seguir en el manual. Tras el apartado introducción (presentación y competencias de la asignatura, estrategias de uso del Texto Guía y criterios de evaluación), los contenidos quedaban organizados en torno a capítulos y dentro de cada uno de ellos se encuentran los siguientes puntos:

\section{MÉTODO}

\subsection{Objetivo}

La experiencia que en este artículo presentamos está enmarcada en un proyecto de innovación docente coordinado por el profesor José Luis Serrano, concedido desde la Convocatoria para promover proyectos y acciones de innovación y mejora en la Universidad de Murcia. Esta convocatoria pretende impulsar iniciativas -en materia de docencia universitaria- relacionadas con la producción e integración de recursos y materiales, promoviendo metodologías docentes que favorezcan un aprendizaje activo, social y participativo.

Uno de los objetivos del proyecto de innovación es evaluar el uso y el impacto del Texto Guía en la asignatura presentada anteriormente. Siguiendo esta dirección, la principal finalidad de este artículo es evaluar el uso de este material docente por parte de los estudiantes según su percepción. En concreto, se pretende recabar información sobre la utilidad del contenido y la estructura del Texto Guía. Además, se analizan las similitudes y diferencias en función del género que pueden influir en el uso del Texto Guía.

Los resultados obtenidos permitirán tomar decisiones al equipo docente para futuras ediciones de este material docente. Además, los resultados aquí presentados podrán ser de utilidad para otros docentes que han creado y utilizado Textos Guías en sus asignaturas, y por supuesto, para el Centro de Formación y Desarrollo Profesional de la Universidad de Murcia, centro que diseña y gestiona cada curso académico la convocatoria Textos Guía. 


\subsection{Contexto}

"Aplicaciones sociales de las TIC para la escuela y el tiempo libre" es el nombre de una asignatura obligatoria de la mención de recursos educativos para la escuela y el tiempo libre del plan de estudios del Grado en Educación Primaria de la Universidad de Murcia. Con un total de 6 créditos ECTS, y según la guía docente ${ }^{1}$, se espera que el alumnado desarrolle competencias para reflexionar y analizar las implicaciones socio-culturales y educativas de las tecnologías en los procesos de acceso, edición y construcción compartida de información en red. Además, se desea que el alumnado sea capaz de diseñar, de manera crítica, acciones formativas destinadas al alumnado de Educación Primaria y a sus familias, tanto en contextos educativos formales como en los no formales.

Los contenidos de la asignatura están distribuidos en cuatro temas, los cuáles son desarrollados durante el primer cuatrimestre de cada curso académico:

- Sociedad, cultura y tecnología es el título del primer tema de la asignatura. Se abordan conceptos clave que permiten al alumnado un mejor seguimiento de la asignatura. Los principales contenidos que se tratan son: ciudadanía digital, cibercultura, seguridad y riesgos asociados a Internet, globalización y brecha digital. Este tema es desarrollado durante las primeras cuatro semanas de la asignatura.

- Gestión del conocimiento y el aprendizaje en un mundo digital. Las actuales visiones del aprendizaje permanente y la revolución tecnológica han propiciado el interés por el desarrollo y estudio de los PLE, siendo un marco eficaz para los aprendizajes a lo largo de toda la vida mediante la creación de comunidades virtuales con el uso del software social. De otro lado, se aborda en este tema la importancia de construir nuestra identidad digital puesto que nos puede ayudar a mejorar nuestras opciones de empleabilidad y minimizar los riesgos que se pueden generar en la red. Como futuros docentes, la configuración de una óptima identidad digital podrá propiciar -además- que sean capaces de reflejar de manera abierta y conectada las tareas que como estudiantes realizan en cada asignatura, demostrando así su especialización en su campo. Para ello se hace necesario conocer las mecanismos, estrategias y herramientas que permiten "aumentar" dichas prácticas docentes e investigadoras. Este tema se desarrolla desde la semana cuatro hasta la semana ocho de la asignatura.

- Diseño y producción de recursos. A lo largo de este tercer tema los estudiantes pudieron analizar los principales conceptos para el diseño y la producción de recursos, tratando contenidos como: materiales didácticos, recursos educativos abiertos, software libre, programación y Scratch, mobile learning y apps para la educación. La duración total de este tema fue de dos semanas.

- Redes de colaboración para la educación informal y promoción comunitaria. Con este tema finaliza la asignatura en lo que a contenidos se refiere. Los principales aspectos tratados son: desarrollo comunitario en la Sociedad Red, redes de colaboración y participación ciudadana, construcción de proyectos de acción comunitaria en el tiempo libre y comunidades de práctica y redes de aprendizaje. La duración prevista para este tema era de dos semanas, sin embargo, debido a diferentes ajustes solamente se pudo hacer uso de una semana.

La dinámica general de la asignatura es la siguiente: el docente realiza una breve exposición de contenido, indica las lecturas clave y la parte del Texto Guía que puede ser de utilidad para clarificar los contenidos abordados. Seguidamente se presenta

${ }^{1}$ Guía Docente de la asignatura disponible en: https://goo.gl/DCy9PE 
una actividad para que, por grupos de 3-5 estudiantes, profundicen en los contenidos y sobre todo conecten la información tratada más a nivel teórico con situaciones o elementos más prácticos. De esta manera, el diseño y la implementación de las actividades se convierten en procesos fundamentales en esta asignatura, puesto que sirven de eslabón entre los contenidos teóricos y las competencias de la asignatura.

Siete fueron las actividades ${ }^{2}$ que los estudiantes tuvieron que realizar por grupos, dos por cada tema salvo en el último tema, puesto que solo fue posible realizar una actividad. Todas las actividades tienen los siguientes apartados: título, finalidad, instrucciones para el alumnado, productos generados por los estudiantes, lecturas clave y rúbrica para la evaluación de la actividad. A continuación, se muestra una breve reseña de cada actividad:

- Actividad 1: Convocatoria abierta Smart Citizen. La finalidad de esta actividad es desarrollar un proyecto de intervención en el tiempo libre en relación con el concepto de Ciudadanía Digital. Para ello, el alumnado cuenta con las bases de una convocatoria real para el desarrollo de proyectos, debiendo ajustarse a la misma.

- Actividad 2: Desenmarañando los riesgos asociados al uso de la red. La finalidad de esta actividad es analizar los riesgos asociados al uso de la red, para ello se diseñará una dinámica grupal cuyo objetivo sea la sensibilización, concienciación y buen uso de la red.

- Actividad 3: Adivina adivinanza. Con esta actividad se analizan los distintos factores que intervienen en la construcción de la identidad digital para mejorar la visibilidad de los riesgos y las ventajas de tener un óptimo desarrollo de la identidad digital.

- Actividad 4: PLE de entrada, procesado y salida. Mediante esta actividad los estudiantes pueden explorar diversas herramientas Web 2.0 que permitirán acceder, filtrar, seleccionar, analizar, compartir y crear conocimiento para la mejora de la competencia investigadora.

- Actividad 5: Creación de una App educativa. Con esta actividad se pretende que los estudiantes puedan crear una aplicación gratuita para móvil partiendo de principios de calidad de diseño de recursos educativos.

- Actividad 6: Programando con Scratch. La finalidad de esta actividad es diseñar y programar un recurso educativo con la herramienta Scratch partiendo de los intereses de los propios estudiantes.

- Actividad 7: Inspiraciones para aprender. Mediante la realización de esta actividad se pretende que el alumnado analice una de las iniciativas de acción comunitaria seleccionadas previamente por el equipo docente.

El sistema de evaluación de la materia está compuesto por dos instrumentos de recogida de información:

- Examen oral (60\% de la calificación final). Los criterios de valoración utilizados son: precisión conceptual, claridad expositiva, estructuración de ideas, espíritu crítico en la presentación y desarrollo de los contenidos.

- Portafolio digital (40\% de la calificación final) que recogerá las tareas realizadas por el alumnado. Los criterios de valoración son: presentación y expresión escrita, dominio de contenidos específicos, gestión de la información, creatividad y espíritu crítico y trabajo grupal.

\footnotetext{
${ }^{2}$ Todas las actividades se encuentran disponibles en: http://ocw.um.es/cc.-sociales/aplicaciones-socialesde-las-tic-para-la-escuela-y/programa
} 


\subsection{Participantes}

El número de matriculados en la asignatura era de 48 estudiantes, de los que 39 respondieron al cuestionario (se presenta con detalle en el apartado siguiente) que se les facilitó una vez finalizada la asignatura. El 55\% son de género femenino y un $45 \%$ de género masculino. El rango de edad de los estudiantes comprende desde los 21 años hasta los 38, estando la inmensa mayoría en el rango de edad de los 21 a los 24 (79\%). El $92 \%$ de los encuestados afirma haber asistido de manera regular a las clases y un $64 \%$ ha utilizado textos guía en otras asignaturas.

\subsection{Instrumento de recogida de información}

Para el proceso de recogida de información, se seleccionó el cuestionario como herramienta pertinente para recabar información rigurosa. Teniendo en cuenta el objetivo de estudio, se hizo necesario diseñar un cuestionario ad hoc. Para ello se siguieron las fases de construcción expuestas por Martín (2011): en primer lugar, se definieron los contenidos que se iban a recoger en el cuestionario, después se concretaron las variables y a partir de ellas se redactaron las preguntas específicas, ordenando las mismas como último paso.

El cuestionario quedó elaborado con 5 preguntas demográficas y con 30 preguntas tipo Likert de 5 opciones englobadas en tres dimensiones (Tabla 1): uso del Texto guía, contenido del Texto guía y actividades.

Tabla 1. Variables y número de ítems por dimensiones del cuestionario

\begin{tabular}{|c|c|c|}
\hline Dimensión & $\mathrm{N}^{\circ}$ de ítem & Variables \\
\hline Datos demográficos & $1,2,3,4,5,6$ & $\begin{array}{l}\text { Sexo, edad, otras titulaciones, } \\
\text { convocatorias en la asignatura y } \\
\text { asistencia regular }\end{array}$ \\
\hline Uso del Texto Guía & $7,8,9,10,11,12$ & $\begin{array}{l}\text { Seguimiento de la asignatura; } \\
\text { lectura de la introducción, } \\
\text { contenidos teóricos, rúbricas de } \\
\text { evaluación de las actividades, } \\
\text { bibliografía de consulta de cada } \\
\text { capítulo y los interrogantes centrales } \\
\text { del tema de cada capítulo }\end{array}$ \\
\hline $\begin{array}{l}\text { Contenido del Texto } \\
\text { Guía }\end{array}$ & $\begin{array}{l}13,14,15,16,17,18,19,20,21 \\
22,23,24,25,26,27,28\end{array}$ & $\begin{array}{l}\text { Apartado introducción, esquemas de } \\
\text { contenido, interrogantes centrales } \\
\text { del tema, contenidos teóricos por } \\
\text { capítulos ( } 4 \text { en total) para preparar } \\
\text { el examen, la realización de las } \\
\text { tareas propuestas y la comprensión } \\
\text { de los conceptos clave }\end{array}$ \\
\hline Actividades & $29,30,31,32,33,34,35$ & $\begin{array}{l}\text { Conexión con la práctica profesional, } \\
\text { comprensión de los contenidos } \\
\text { teóricos de cada capítulo, trabajo en } \\
\text { equipo, rúbricas de evaluación como } \\
\text { punto de partida, comprensión de } \\
\text { las instrucciones, herramienta Blog, } \\
\text { relación entre finalidad de la } \\
\text { actividad y su comprensión }\end{array}$ \\
\hline
\end{tabular}

Como primera fase de validación de contenido del cuestionario se utilizó la técnica de juicio de expertos. Se seleccionaron ocho expertos: dos de ellos especialistas en métodos de investigación y análisis de datos, cinco expertos en el área de Didáctica y Organización Escolar, teniendo uno de ellos experiencia en la elaboración de textos guía en la Universidad de Murcia. Se preparó una hoja con las instrucciones y la plantilla a rellenar, en la que se preguntaba por la coherencia, relevancia y claridad de cada ítem y la suficiencia de la dimensión (Escobar-Pérez y Cuervo-Martínez, 2008), y 
se mandó a los 8 expertos.

Una vez recogidas todas las plantillas de los expertos se procedió al análisis de los datos obtenidos. Se decidió eliminar aquellos ítems que obtuvieron una puntuación inferior a 2 y/o una desviación típica superior a 1, salvo en aquellos casos en los que los jueces aconsejaron su reelaboración (Crocker y Algina, 1986).

Se aplicaron los 29 ítems a la muestra de conveniencia y se realizó un análisis de ítems que derivó en una escala final de 26 ítems cuyos índices de homogeneidad fueron mayores de .30. La fiabilidad global del instrumento ${ }^{3}$ al finalizar todo el proceso fue de .943 .

\section{RESULTADOS}

Esta investigación se podría incluir como estudio exploratorio combinado con un análisis descriptivo. En este tipo de estudios, considerado como inicial a falta de trabajos previos, se pretende hacer una representación de la realidad y generar ideas para ser estudiadas a otros niveles. Por lo tanto, en coherencia con el tipo de investigación planteada, se ha realizado un análisis de datos de tipo descriptivo realizado con el programa estadístico SPSS Statistics 22 para Windows, obteniendo resultados a través de las operaciones propias de la estadística descriptiva (frecuencias, porcentajes, media, mediana y moda).

Siguiendo las recomendaciones de algunos autores (Cubo, 2011; Pardo, Ruiz, y SanMartín, 2015) no se han realizado pruebas paramétricas teniendo en cuenta la representatividad de la muestra, en este caso una muestra por conveniencia ya que es la población diana del Texto Guía creado, utilizado y evaluado.

Presentamos a continuación los resultados más relevantes organizados en tres grandes bloques: uso y lectura de los distintos apartados; utilidad del contenido; información referente a las actividades realizadas. Además, indicaremos las diferencias significativas en relación al género en cada caso.

\subsection{Cuestiones relativas al uso y lectura de los distintos apartados del Texto guía}

El 76.9\% del alumnado afirma haber usado el Texto Guía para seguir la asignatura, mientras que el $7.7 \%$ de estudiantes afirma no haberlo usado. Dentro de este apartado existen diferencias en cuanto al género ya que un $90.5 \%$ de las mujeres responde estar de acuerdo y totalmente de acuerdo con esta afirmación frente al 58.8\% de los hombres.

A continuación, se hace referencia a la lectura de la introducción al Texto guía y de los distintos apartados que conformaban cada capítulo, contenidos teóricos, rúbricas de evaluación, bibliografía de consulta e interrogantes centrales del tema.

Lectura del apartado "Introducción al Texto guía": el 48.7\% de los estudiantes afirma estar totalmente de acuerdo y de acuerdo con respecto a haber leído este apartado, mientras que el $33.3 \%$ asegura no haberlo hecho. En este sentido, el porcentaje en el grado de desacuerdo y totalmente en desacuerdo es mayor en hombres $(41.1 \%)$ que en mujeres $(23.8 \%)$.

Lectura de los contenidos teóricos de cada capítulo: dentro de este apartado se eleva el porcentaje de los estudiantes que afirman estar totalmente de acuerdo y de acuerdo en haber leído este apartado, llegando al 79,5\% frente al $20.5 \%$ de alumnos que afirmaban estar totalmente en desacuerdo y en desacuerdo con esta afirmación.

Lectura de las rúbricas de evaluación de las actividades: el 60.9\% de los estudiantes afirma haber leído las rúbricas de evaluación frente al $25.6 \%$ que afirma estar totalmente en desacuerdo y en desacuerdo con respecto a la afirmación sobre la lectura de

\footnotetext{
${ }^{3}$ Acceso a la versión final del instrumento en modo previsualización: https://encuestas.um.es/MTQyMD I.w
} 
este apartado del Texto guía.

Dentro de este apartado encontramos bastante diferencia ya que el $47.6 \%$ de las mujeres responden estar de acuerdo frente al $35.3 \%$ de los hombres, siendo la diferencia aún mayor si sumamos los porcentajes de respuestas de totalmente de acuerdo y de acuerdo, $66.6 \%$ en las mujeres y $47.1 \%$ en los hombres.

Lectura de la bibliografía de consulta de cada capítulo: dentro de este apartado tenemos que destacar el alto porcentaje de alumnos que se queda en el rango intermedio de la escala, puesto que el $30.8 \%$ responde ni acuerdo ni en desacuerdo con respecto a la lectura de este apartado, siendo incluso superior al porcentaje de alumnos que responden estar totalmente de acuerdo y de acuerdo (28.2\%). Además, el $41 \%$ del alumnado afirma estar totalmente en desacuerdo y en desacuerdo. Con respecto a las diferencias de género podemos afirmar que responden a los datos generales, siendo menor el porcentaje en hombres que parece afirmar haber leído la bibliografía de consulta. La mayor diferencia la encontramos en la respuesta intermedia "ni acuerdo ni en desacuerdo" (19\% de respuestas del género femenino frente al $41.2 \%$ de respuestas de los hombres).

Lectura de los interrogantes centrales del tema: el 66.7\% afirma estar totalmente de acuerdo y de acuerdo con la afirmación relativa a la lectura de este apartado siendo solamente el $7.7 \%$ los que responden estar en desacuerdo y totalmente en desacuerdo con esta afirmación.

Dentro de las diferencias encontradas por género destacamos que ninguno de los hombres encuestados responde los grados de totalmente en desacuerdo ni en desacuerdo frente al $14.3 \%$ de las mujeres que contestaron estas dos opciones.

\subsection{Utilidad de distintos apartados en los que se divide cada uno de los capítulos}

Las afirmaciones que hacen referencia tanto a los esquemas de contenido como a los interrogantes centrales del tema aparecen con porcentajes altos en los grados de totalmente de acuerdo y de acuerdo (73.7\% esquemas de contenido y 75.7\% interrogantes centrales). El apartado de introducción obtiene un 59\% de respuestas que están totalmente de acuerdo y de acuerdo con la afirmación sobre la utilidad de este apartado. El apartado que menor porcentaje recibe en los grados de totalmente de acuerdo y de acuerdo es el de utilidad de la bibliografía de consulta con un $43.4 \%$, dato que concuerda con los datos que hacen referencia a la lectura de este apartado.

Estos mismos datos se repiten cuando los analizamos en función del género, encontrando la mayor diferencia en la afirmación relativa a los interrogantes centrales del tema, ya que $84.2 \%$ de las mujeres responden estar totalmente de acuerdo y de acuerdo con esta afirmación frente al $70.6 \%$ de los hombres.

A continuación se indican los principales datos obtenidos en relación con la utilidad de los contenidos de cada uno de los capítulos relacionados con tres aspectos: preparación del examen, realización de las tareas y comprensión de conceptos clave.

Capítulo 1: Las afirmaciones en sentido positivo en los tres aspectos están por encima en todos los casos del 53\%, siendo la utilidad con relación a la realización de las tareas como la comprensión de los conceptos clave del tema los que mayor grado de acuerdo cuentan $(66.7 \%$ y $66.7 \%$, respectivamente).

Capítulo 2: dentro de este capítulo encontramos que los grados de acuerdo y totalmente de acuerdo también están por encima del 53\% en los tres aspectos contemplados, en esta ocasión la utilidad para preparar el examen y para la comprensión de conceptos clave son las afirmaciones que más porcentajes de acuerdo tienen $(65.8 \%$ y $70.3 \%)$.

Capítulo 3: en este capítulo los tres aspectos aparecen con valoraciones con respecto a los grados de acuerdo y totalmente de acuerdo por encima del 55\% siendo la utilidad con relación a la realización de las tareas y a la comprensión de conceptos clave los mejor valorados $(64.1 \%$ y $61.5 \%)$. 
Capítulo 4: los porcentajes de totalmente de acuerdo y de acuerdo en los tres apartados dentro de este capítulo están por encima del $56 \%$ siendo la utilidad para preparar el examen y para comprender los conceptos clave los que mayor porcentaje reciben, en ambos casos un $60.5 \%$.

En la Tabla 2 aparecen los datos relativos a los porcentajes de respuesta diferenciados por sexo. Resaltamos a este respecto la diferencia existente con respecto a la afirmación que hace referencia a los contenidos del tema 4 y su utilidad para comprender los conceptos clave.

Tabla 2. Utilidad de los diferentes contenidos del Texto Guía

\begin{tabular}{|c|c|c|c|c|c|}
\hline & & \multicolumn{2}{|c|}{$\begin{array}{c}\text { Totalmente de acuerdo / } \\
\text { De acuerdo }\end{array}$} & \multicolumn{2}{|c|}{$\begin{array}{c}\text { Totalmente en } \\
\text { desacuerdo / En } \\
\text { desacuerdo }\end{array}$} \\
\hline & & Mujeres & Hombres & Mujeres & Hombres \\
\hline \multirow{3}{*}{$\begin{array}{l}\text { Capítulo } \\
1\end{array}$} & $\begin{array}{l}\text { Los contenidos me han sido útiles } \\
\text { para preparar el examen }\end{array}$ & $57.1 \%$ & $52.9 \%$ & $9.5 \%$ & $5.9 \%$ \\
\hline & $\begin{array}{l}\text { Los contenidos me han sido útiles } \\
\text { para realizar las tareas }\end{array}$ & $71.4 \%$ & $58.8 \%$ & $4.8 \%$ & $5.9 \%$ \\
\hline & $\begin{array}{l}\text { Los contenidos me han sido útiles } \\
\text { para comprender los conceptos clave }\end{array}$ & $71.4 \%$ & $58.8 \%$ & $4.8 \%$ & $11.8 \%$ \\
\hline \multirow{3}{*}{$\begin{array}{l}\text { Capítulo } \\
2\end{array}$} & $\begin{array}{l}\text { Los contenidos me han sido útiles } \\
\text { para preparar el examen }\end{array}$ & $71.4 \%$ & $62.6 \%$ & $14.3 \%$ & $6.3 \%$ \\
\hline & $\begin{array}{l}\text { Los contenidos me han sido útiles } \\
\text { para realizar las tareas }\end{array}$ & $75 \%$ & $64.7 \%$ & $10 \%$ & $0 \%$ \\
\hline & $\begin{array}{l}\text { Los contenidos me han sido útiles } \\
\text { para comprender los conceptos clave }\end{array}$ & $79 \%$ & $64.7 \%$ & $10.6 \%$ & $11.8 \%$ \\
\hline \multirow{3}{*}{$\begin{array}{l}\text { Capítulo } \\
3\end{array}$} & $\begin{array}{l}\text { Los contenidos me han sido útiles } \\
\text { para preparar el examen }\end{array}$ & $60 \%$ & $52.9 \%$ & $10 \%$ & $0 \%$ \\
\hline & $\begin{array}{l}\text { Los contenidos me han sido útiles } \\
\text { para realizar las tareas }\end{array}$ & $76.2 \%$ & $52.9 \%$ & $4.8 \%$ & $11.8 \%$ \\
\hline & $\begin{array}{l}\text { Los contenidos me han sido útiles } \\
\text { para comprender los conceptos clave }\end{array}$ & $71.4 \%$ & $47 \%$ & $4.8 \%$ & $17.7 \%$ \\
\hline \multirow{3}{*}{$\begin{array}{l}\text { Capítulo } \\
4\end{array}$} & $\begin{array}{l}\text { Los contenidos me han sido útiles } \\
\text { para preparar el examen }\end{array}$ & $71.4 \%$ & $50.1 \%$ & $14.3 \%$ & $18.8 \%$ \\
\hline & $\begin{array}{l}\text { Los contenidos me han sido útiles } \\
\text { para realizar las tareas }\end{array}$ & $71.4 \%$ & $41.1 \%$ & $14.3 \%$ & $17.6 \%$ \\
\hline & $\begin{array}{l}\text { Los contenidos me han sido útiles } \\
\text { para comprender los conceptos clave }\end{array}$ & $85 \%$ & $35.3 \%$ & $5 \%$ & $35.3 \%$ \\
\hline
\end{tabular}

\subsection{Algunos datos referentes a las actividades realizadas}

En general todos los aspectos preguntados en relación con las actividades aparecen con porcentajes muy altos en los grados de totalmente de acuerdo y de acuerdo. La afirmación "la herramienta Blog me parece adecuada como modo de entrega de las actividades" la que mayor porcentaje recibe con un $89.7 \%$ en los grados de totalmente de acuerdo / de acuerdo. Por el contrario, la afirmación que hace referencia al uso de las rúbricas como punto de partida para la realización de las actividades, es la que tiene menor porcentaje en los grados anteriormente comentados, un $59 \%$.

En la Tabla 3 podemos encontrar un resumen de este apartado.

Tabla 3. Valoración de las actividades realizadas 


\begin{tabular}{|c|c|c|}
\hline \multicolumn{3}{|l|}{ Table 3 continued } \\
\hline & $\begin{array}{l}\text { Totalmente de acuerdo / De } \\
\text { acuerdo }\end{array}$ & $\begin{array}{l}\text { Totalmente en } \\
\text { desacuerdo / en } \\
\text { desacuerdo }\end{array}$ \\
\hline $\begin{array}{l}\text { Las actividades realizadas conectan con } \mathrm{mi} \\
\text { práctica profesional }\end{array}$ & $79.5 \%$ & $5.1 \%$ \\
\hline $\begin{array}{l}\text { Las actividades realizadas facilitan la } \\
\text { comprensión de los contenidos de cada } \\
\text { capítulo }\end{array}$ & $87.2 \%$ & $2.6 \%$ \\
\hline $\begin{array}{l}\text { Las actividades realizadas favorecen el } \\
\text { trabajo en equipo }\end{array}$ & $84.6 \%$ & $7.7 \%$ \\
\hline $\begin{array}{l}\text { He utilizado las rúbricas de evaluación como } \\
\text { punto de partida para la realización de las } \\
\text { actividades }\end{array}$ & $59 \%$ & $23.1 \%$ \\
\hline $\begin{array}{l}\text { He entendido lo que se me pide en cada } \\
\text { actividad con la lectura de las instrucciones }\end{array}$ & $77 \%$ & $5.1 \%$ \\
\hline $\begin{array}{l}\text { La herramienta Blog me parece adecuada } \\
\text { como modo de entrega de cada actividad }\end{array}$ & $89.7 \%$ & $2.6 \%$ \\
\hline $\begin{array}{l}\text { El apartado final de la actividad me ha } \\
\text { ayudado a comprender qué tengo que hacer } \\
\text { en cada actividad }\end{array}$ & $84.6 \%$ & $2.6 \%$ \\
\hline
\end{tabular}

Los datos analizados desde la perspectiva de género no reflejan grandes diferencias salvo en los ítems referidos a la lectura de las instrucciones. Los hombres que responden estar totalmente de acuerdo y de acuerdo con esta afirmación es algo mayor que el de las mujeres (82.3\% frente al 71.4\%). En el caso de la utilización de las rúbricas de evaluación como punto de partida para la realización de las actividades son las mujeres las que en un mayor porcentaje responden estar de totalmente de acuerdo y de acuerdo con esta afirmación ( $66.7 \%$ frente $47 \%)$.

\section{CONCLUSIONES}

La integración del Texto Guía en la asignatura "Aplicaciones sociales de las TIC para la escuela y el tiempo libre" requería de un análisis que posibilite una verdadera comprensión del impacto que, según los estudiantes, tiene en el proceso de enseñanza y aprendizaje. Los datos, por tanto, reflejan una serie de aspectos que nos ayudarán a mejorar el diseño de este material docente y, en consecuencia, basar parte de nuestras funciones como docentes universitarios en evidencias. De otro lado, este trabajo también puede contribuir al enriquecimiento de la convocatoria de Proyectos Textos Guía que publica cada año la Universidad de Murcia ya que se ha analizado la estructura de este material, el cual viene predefinida en la propia convocatoria.

De forma general, la utilidad del Texto Guía es valorada de forma positiva por parte del alumnado de la asignatura, aunque la propia explicación del Texto Guía fue uno de los apartados menos leídos y de menos utilidad según los estudiantes participantes. Esto podría deberse al gran pragmatismo seguido por el alumnado con el uso de este material puesto que es muy utilizado para preparar el examen y realizar las tareas de la asignatura.

La inclusión de las rúbricas de evaluación en cada tarea no ha sido valorado positivamente. En realidad, los estudiantes no las han utilizado. De esta manera han realizado las actividades sin consultar previamente estos instrumentos de evaluación desaprovechando sus virtudes. Los datos obtenidos nos invitan a concluir que existe la necesidad de añadir un capítulo que explique la importancia que tienen las rúbricas en el proceso de aprendizaje de nuestro alumnado, ya que como observamos en los 
resultados presentados anteriormente, este es otro de los puntos que menores porcentajes recibe con respecto a su lectura y utilidad. De forma complementaria, podría ser conveniente el uso de la propuesta metodológica presentada por la Cruz Flores y Hernández (2014) para la incorporación de rúbricas de evaluación. Incluso, como indican Berrocoso y Gómez (2014), es necesario formar a los estudiantes para el uso de estos instrumentos.

También vemos necesario revisar, como se decía al inicio de este artículo, la importancia y la forma de presentar la bibliografía dentro del Texto Guía, puesto que al igual que ocurre con las rúbricas es otro aspecto que los datos nos sugieren reflexionar ya que no ha sido consultada.

La estructura planteada dentro de los capítulos, así como el contenido de cada uno de ellos, parece tener una buena aceptación entre el alumnado a la luz de los datos obtenidos con respecto a su utilidad y su relación con la preparación del examen, la realización de tareas y la comprensión de conceptos clave. Desde nuestro punto de vista, la utilidad con respecto a la comprensión de conceptos -es uno de los aspectos mejor valorados en cada uno de los capítulos- tiene mucha relación con los otros dos aspectos valorados, por lo que creemos que es importante seguir manteniendo la estructura planteada en el Texto Guía.

El blog también resultó una herramienta adecuada para la entrega de tareas para el alumnado participante, siendo lo mejor valorado de todo el proceso. Igualmente, es destacable que los alumnos consideren las actividades planteadas en la asignatura como favorecedoras del trabajo en equipo.

El hecho de que el uso de la bibliografía complementaria no sea de utilidad para el alumnado concuerda con las ideas previas establecidas de que no contrasta la información aportada por el profesor (Gutierrez et al., 2017).

Es interesante, tal y como reflejan los datos, reflexionar en torno a las diferencias encontradas entre las mujeres y los hombres con respecto a la utilidad de los capítulos tres y cuatro, durante la realización de las tareas y la comprensión de conceptos clave, siendo mayor -la utilidad percibida- en el caso de las mujeres. Estos datos nos llevan a reflexionar si la propia temporalización de la asignatura ha influido en estas valoraciones, ya que en el caso de estos capítulos la dedicación en las clases presenciales fue algo menor, aunque esta relación entre tiempos y utilidad de los capítulos no fue establecida en el cuestionario.

Otra de las diferencias con respecto al género es la encontrada en relación con la utilidad de las rúbricas. Nos podría indicar que, en el caso de nuestro alumnado del Grado en Educación Primaria, son las mujeres las que mayor importancia le dan al proceso de evaluación. Sin embargo, este tipo conclusiones requerirían un mayor tratado de los datos obtenidos que no se corresponden con los objetivos planteados en esta primera aproximación a los datos. Creemos que podríamos seguir profundizando en futuros trabajos en relación con estas diferencias encontradas en relación con el género. En este caso, es necesario contemplar otras variables que puedan estar influyendo y utilizar una muestra mayor.

No se han encontrado estudios similares que analicen las diferencias de uso de materiales docentes en función del género y que nos permitan discutir nuestros resultados. Conocer este tipo de diferencias puede aportar información clave para la definición de criterios objetivos en la configuración de los equipos de trabajo para la realización de las actividades, por ejemplo.

Para concluir, afirmamos que el Texto Guía que hemos diseñado y utilizado durante la asignatura es un material docente que es valorado de forma positiva en su uso y utilidad. La estructura que sigue es también valorada adecuadamente por el alumnado. Lo mismo sucede con los apartados y capítulos en los que se divide, existiendo una buena relación entre estos aspectos y los objetivos tanto de la asignatura como los objetivos de aprendizaje del conjunto del alumnado, la cual podrá ser objeto central 
de análisis en futuros estudios. Dicho de otro modo, analizar de manera rigurosa la metodología general seguida en la asignatura y la integración del Texto Guía. Esto nos permitirá poder confirmar algunas de las hipótesis que este estudio nos ha invitado a generar.

\section{REFERENCIAS}

Berrocoso, J. V., y Gómez, A. C. (2014). El uso de e-rúbricas para la evaluación de competencias en estudiantes universitarios. Estudio sobre fiabilidad del instrumento. REDU. Revista de Docencia Universitaria, 12(1), 49-79.

Carlino, P. (2003). Leer textos complejos al comienzo de la educación superior: tres situaciones didácticas para afrontar el dilema. Textos. Didáctica de la Lengua y la Literatura(33), 43-51. Descargado de https://www.aacademica.org/paula.carlino/ 172.pdf

Crocker, L., y Algina, J. (1986). An introduction to classical and modern test theory. New York: Holt, Rinehart \& Winston.

Cubo, S. (2011). Muestreo. En S. Cubo, B. Marín, y J. L. G. Ramos (Eds.), Métodos de investigación y análisis de datos en ciencias sociales y de la salud (pp. 110-133). Madrid: Ediciones Pirámide.

Egaña, T., Bidegain, E., y Zuberogoitia, A. (2013). ¿Cómo buscan información académica en internet los estudiantes universitarios? Lo que dicen los estudiantes y sus profesores. Edutec.Revista electrónica de tecnología educativa, 43, 1-15. https://doi.org/10.21556/ edutec.2013.43.332

Escobar-Pérez, J., y Cuervo-Martínez, A. (2008). Validez de contenido y juicio de expertos: una aproximación a su utilización. Avances en medición, 6, 27-36. Descargado de http://www.humanas.unal.edu.co/psicometria/files/7113/8574/5708/Articulo3_Juicio _de_expertos_27-36.pdf

Estienne, V., y Carlino, P. (2004). (Vol. 4). Descargado de http://aprendeenlinea.udea.edu.co/ revistas/index.php/unip/issue/current

Gutierrez, I., Lopéz, P., Serrano, J. L., y Solano, I. M. (2017). Estrategias y herramientas para aprender: descripción de los PLE de los estudiantes universitarios españoles y propuestas para la mejora de la respuesta institucional. En M. P. Prendes y M. M. Román (Eds.), Entornos personales de aprendizaje: una visión actual de cómo aprender con tecnologías (pp. 65-88). Barcelona: Octaedro.

Gutiérrez, I., y Serrano, J. L. (2016). Evaluation and development of digital competence in future primary school teachers at the University of Murcia. fournal of New Approaches in Educational Research, 5(1), 51-56. https://doi.org/10.7821/naer.2016.1.152

Henry, L. A. (2006). SEARCHing for an Answer:The critical role of new literacies while reading on the Internet. The reading teacher, 59(7), 614-627. https://doi.org/10.1598/ RT.59.7.1

la Cruz Flores, G. D., y Hernández, L. F. A. (2014). Rúbricas y autorregulación: pautas para promover una cultura de la autonomía en la formación profesional. REDU. Revista de Docencia Universitaria, 12(1), 31-48.

López-Flamarique, P. (2017). Buscar, evaluar y seleccionar información digital: una experiencia didáctica en Educación Secundaria Obligatoria (Tesis Doctoral no publicada). Universidad de Mondragón.

Marín, M. (2006). Alfabetización académica temprana. Lectura y vida. Revista latinoamericana de lectura, 27(4), 30-47. Descargado de http://www.lecturayvida.fahce.unlp.edu.ar/ numeros/a27n4/27_04_MarIn.pdf

Martín, B. (2011). Técnicas e instrumentos de recogida de investigación. En B. Martín y J. L. Ramos (Eds.), Métodos de investigación y análisis de datos en ciencias sociales y de la salud (pp. 173-233). Pirámide.

Marucco, M. (2011). ¿Por qué los docentes universitarios debemos enseñar a leer y a escribir 
a nuestros alumnos? Revista Electrónica de Didáctica en Educación Superior(2), 1-7. Descargado de https://isfd87-bue.infd.edu.ar/sitio/upload/Marucco.pdf

Pardo, A., Ruiz, M. A., y San-Martín, R. Y. (2015). Análisis de datos en ciencias sociales y de la salud I. Madrid: Síntesis.

Peña, F. J. (2011). Leer y escribir. Prácticas necesarias en la universidad. Educere, 15(52), 711-719. Descargado de https://www.redalyc.org/pdf/356/35622379018.pdf

Peña, L. B. (2008). La competencia oral y escrita en la educación superior. Descargado de http://www.mineducacion.gov.co/1621/articles-189357_archivo_pdf_comunicacion .pdf

Prendes, M. P., Solano, I. M., Serrano, J. L., González, V., y Román, M. M. (2018). Entornos Personales de Aprendizaje para la comprensión y desarrollo de la Competencia Digital: un estudio exploratorio con futuros profesionales. Revista Educatio XXI, 36(2), 115-134. https://doi.org/10.6018/j/333081

Serrano, J. L., Román, M. M., González, V., y Prendes, M. P. (2016). Aplicaciones sociales de las TIC para la Escuela y el tiempo libre. Murcia: Editum.

Vázquez, A. (2005). ¿Alfabetización en la Universidad? . Re-conociendo los problemas educativos en la Universidad. Colección de Cuadernillos de actualización para pensar la enseñanza universitaria. Argentina. Descargado de https://www.unrc.edu.ar/unrc/ academica/docs/publicaciones/cuadernillo01.pdf 